\title{
Use of heated humidification during nasal CPAP titration in obstructive sleep apnoea
} syndrome

\author{
M. Duong*,\# , L. Jayaram*, D. Camfferman*, P. Catcheside*, \\ I. Mykytyn* and R.D. McEvoy*
}

ABSTRACT: Nasal symptoms associated with the use of nasal continuous positive airway pressure (nCPAP) in obstructive sleep apnoea (OSA) can adversely impact on patients' tolerance, acceptance and adherence to nCPAP therapy. Regular use of heated humidification is effective in alleviating these symptoms and improve patient comfort.

In a randomised, parallel, double-blinded, controlled study, the present authors examined the use of heated humidification during a single night laboratory nCPAP titration in untreated OSA patients and its effect on nasal symptoms, nasal airway resistance (NAR), effective pressure and treatment tolerability and acceptance.

Baseline characteristics of subjects $(n=70)$ receiving placebo and humidification were (mean $\pm \mathrm{SEM}$ ): age $51.2 \pm 2.2$ versus $50.6 \pm 1.6 \mathrm{yrs}$; body mass index $33.6 \pm 0.9$ versus $35.2 \pm 0.9 \mathrm{~kg} \cdot \mathrm{m}^{-2}$; Epworth Sleepiness Scale 10.8 \pm 1.0 versus $11.3 \pm 0.7$; and apnoea-hypopnoea index $43.5 \pm 4.6$ versus $44.4 \pm 4.1$ events $\cdot h^{-1}$. Total inspiratory NAR, before $(0.36 \pm 0.09$ (placebo) versus $\left.0.33 \pm 0.09 \mathrm{kPa} \cdot \mathrm{L}^{-1} \cdot \mathrm{s}^{-1}\right)$ and after $\mathrm{nCPAP}\left(0.47 \pm 0.11\right.$ versus $\left.0.29 \pm 0.04 \mathrm{kPa} \cdot \mathrm{L}^{-1} \cdot \mathrm{s}^{-1}\right)$ were not significantly different between the groups. No difference was found in the frequency and severity of nasopharyngeal symptoms, therapeutic pressure and subjective response to nCPAP.

In conclusion, heated humidification during the initial nasal continuous positive airway pressure titration offers no additional benefit in nasal physiology, symptoms or subjective response to nasal continuous positive airway pressure, and, therefore, should not be routinely recommended.

KEYWORDS: Continuous positive airway pressure titration, heated humidification, nasopharyngeal side-effects, obstructive sleep apnoea syndrome, rhinomanometry, upper airway

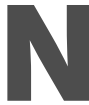

asal continuous positive airway pressure (nCPAP) is currently the treatment of choice for symptomatic obstructive sleep apnoea syndrome (OSAS). Its effectiveness has been well documented [1-4], but the rate of acceptance and long-term adherence to this treatment remain suboptimal [5, 6]; namely, being compromised by unwanted psychosocial and physical side-effects [7-10].

Adverse symptoms of the upper airway are commonly reported with nCPAP $[10,11]$, which can be alleviated with the regular use of heated humidification [12-14]. Under experimental conditions, the administration of nCPAP in the presence of mouth leaks leads to the appearance of nasopharyngeal symptoms and a rise in nasal airway resistance (NAR). The latter was attenuated with heated humidification and is thought to form the physiological basis for the improvement in symptoms and compliance rates observed in clinical studies [15].
Since the early experience with nCPAP may influence acceptance and long-term adherence $[3,16-18]$, the aim of the present study was to investigate the benefit of adding heated humidification to nCPAP on the first night of titration. The current authors hypothesised that humidification would prevent a rise in NAR, significantly reduce the incidence and severity of nasal symptoms, and improve the efficiency of CPAP delivery to the collapsible (pharyngeal) airway segment, thereby lowering the therapeutic positive pressure required. As a result of these mechanisms, heated humidification during the first night might promote increased initial acceptance and potentially future adherence to therapy. Therefore, in a parallel, randomised, placebo-controlled, doubleblinded study, the effect of heated humidification on: 1) total NAR; 2) effective CPAP; 3) the frequency and severity of nasopharyngeal symptoms; and 4) nCPAP tolerability and predicted future use were assessed.
AFFILIATIONS

*Adelaide Institute for Sleep Health South Australia, Australia and \#McMaster University, Hamilton Ontario, Canada.

CORRESPONDENCE

M. Duong

McMaster University

Health Science Centre

Room 3U24

Cardiorespiratory Research Unit

1200 Main Street West

Hamilton

Ontario L8N 375

Canada

Fax: 19055408803

E-mail: duongmy@mcmaster.ca

Received:

November 162004

Accepted after revision:

June 172005 


\section{METHODS \\ Study design}

A total of 70 untreated OSAS patients (aged $\geqslant 18$ yrs) referred for a full overnight polysomnographic nCPAP titration were consecutively enrolled during the year 2001. The exclusion criteria were: 1) significant psychiatric, respiratory and cardiovascular co-morbidities; 2) supplementary oxygen; 3) significant nasal obstruction precluding nCPAP use; 4) previous surgical intervention for OSAS; and 5) inability to provide informed written consent. On the night of the titration, Epworth Sleepiness Scale (ESS) and NAR measurements were obtained. Randomisation to active (Sullivan HumidAire; Resmed, Sydney, Australia) or placebo treatment (HumidAire with a disabled heater, a perspex baffle with equivalent dead space, without water and a cover to mask the humidifier contents) was performed by a daytime technician who did not communicate between subjects or overnight staff. Humidifiers were connected to nCPAP (Sullivan Elite; Resmed) according to the manufacturer's recommendations and administered via a fitted Mirage nasal mask (Res Med Ltd, North Ryde, NSW, Australia). The humidifier setting was routinely set at six (out of eight), which in the experience of the present authors provided the optimal level of humidification without significant condensation. The following morning, NAR measurements were repeated and subjects filled out a questionnaire. The study was approved by the Repatriation General Hospital Ethics Committee, South Australia.

\section{CPAP titration}

The Sleepwatch (Compumedics, Melbourne, Australia) system was used to record electroencephalogram (EEG) $\left(\mathrm{C}_{4} \mathrm{~A}_{1} / \mathrm{C}_{3} \mathrm{~A}_{2}\right)$, bilateral electro-oculogram, submental electromyogram, nasal airflow (pressure transducer), body position (mercury switch), and leg movements (piezo-electric sensors). ECG, oxygen saturation (OxiRadometer; Radiometer, Copenhagen, Denmark), mask pressure, ribcage and abdominal movements by respiratory inductance plethysmography were also monitored. Sleep stages were manually scored according to RECHTSCHAFFEN and KALES [19]. Obstructive apnoea was defined as $<20 \%$ airflow lasting $\geqslant 10 \mathrm{~s}$ with ongoing respiratory efforts. Hypopnoea was defined as $>50 \%$ diminution in the amplitude of two out of three respiratory channels (airflow, thoracic and abdominal) lasting $\geqslant 10 \mathrm{~s}$. The severity of OSAS was expressed as the total apnoea and hypopnoea events per hour of sleep time. Overnight titration was performed by a sleep technologist. Starting with the lowest tolerated pressure $\left(4 \mathrm{cmH}_{2} \mathrm{O}\right)$, increments of $1 \mathrm{cmH}_{2} \mathrm{O}$ were added for obstructive apnoea events or hypopnoea preceding EEG arousals. Subjects had to lie on their back for at least one rapid eye movement (REM) period during the study. An effective CPAP was reached when respiratory events, desaturation and respiratory event-related arousals were eliminated. Mouth leaks were monitored for by close observation for mouth opening, expiratory mouth air venting on video monitoring or spontaneous fluctuations (fall) in baseline CPAP level. If any of these changes occurred, a chinstrap was applied. The therapeutic CPAP was determined by a sleep physician (L. Jayaram) based on the pressure that controlled obstructive events during supine REM sleep. If supine REM sleep was not sampled, the pressure required to control respiratory events during nonsupine REM sleep was determined. If obstructive events were not completely controlled during REM sleep, $2 \mathrm{cmH}_{2} \mathrm{O}$ was added to the final pressure. In instances where the CPAP that controlled respiratory events during REM sleep did not exceed that for non-REM sleep, then the therapeutic non-REM CPAP level was recommended.

\section{Questionnaire}

A questionnaire was used to document baseline characteristics and the subjective responses following overnight nCPAP. Subjects were asked to rate: 1) their quality of sleep; 2) the feeling of morning freshness $(1=$ worse, $2=$ same and $3=$ better than usual); 3) nCPAP tolerability ( $1=$ poor, $2=$ fair, $3=$ good and $4=$ very well); 4 ) predicted future use $(1=$ refuse, $2=$ intermittent, $3=$ frequent and $4=$ all the time); and 5) the occurrence and severity of nasal side-effects (nasal stuffiness, discharge, burning, sore throat and dry mouth) on a $10 \mathrm{~cm}$ visual analogue scale (VAS).

\section{Nasal airway resistance}

Anterior active rhinomanometry (Masterscope Rhino; Jaeger, Hoechberg, Germany) was performed with a face-mask in accordance with the International Committee on Objective Assessment of the Nasal Airways [20]. Measurements were taken after $15 \mathrm{~min}$ in the seated and supine positions during quiet breathing with mouth closed. A minimum of three reproducible curves were recorded from each nostril and the average curve taken as representative. Inspiratory and expiratory NAR obtained from each nostril at a transnasal pressure of $150 \mathrm{~Pa}$ were used to calculate the total nasal resistance (RTotal):

$$
1 / R_{\text {Total }}=1 / R_{\mathrm{L}}+1 / R_{\mathrm{R}}
$$

$R L$ and $R R$ are the left and right nasal resistances, respectively.

\section{Statistical analysis}

Data are expressed as mean \pm SEM. Log transformation of data, which were not normally distributed, was performed prior to the analysis. Comparisons between group means were performed with the unpaired t-test and between proportions by the Chi-squared statistic. A two-tailed p-value $<0.05$ was accepted as significant.

\section{RESULTS}

Baseline characteristics were similarly distributed between groups (table 1). Up to half of the subjects reported allergic rhinitis with similar proportions admitted to using nasal corticosteroids in the previous 12 months. During this period, the most frequently reported nasosinus symptom was nasal stuffiness, followed by sore throat (23-28\%) and runny nose (7-17\%).

Two subjects (one placebo, one humidification) were intolerant to $\mathrm{nCPAP}$ and titration was stopped within $2 \mathrm{~h}$ of commencing therapy due to claustrophobia from the mask. Their data were excluded from subsequent analysis.

\section{Nasal airway resistance}

A total of $48(70.6 \%)$ subjects had technically acceptable NAR data (i.e. free from nonzero baseline offset, and inspiratory and expiratory transnasal pressures achieved the requisite $150 \mathrm{~Pa}$ in both nostrils). Comparisons of baseline characteristics and outcome variables between subjects, with and without missing 


\begin{tabular}{|c|c|c|}
\hline Variables & Placebo & $\begin{array}{c}\text { Heated } \\
\text { humidification }\end{array}$ \\
\hline Subjects $\mathrm{n}$ & 36 & 34 \\
\hline Sex M:F & $34: 2$ & $28: 6$ \\
\hline Age yrs & $51.2 \pm 2.2(20-72)$ & $50.6 \pm 1.6(28-67)$ \\
\hline BMI $\mathbf{k g} \cdot \mathbf{m}^{-2}$ & $33.6 \pm 0.9(25.3-44.6)$ & $35.2 \pm 0.9(27.2-48.7)$ \\
\hline ESS & $10.8 \pm 1.0(0-23)$ & $11.3 \pm 0.7(3-19)$ \\
\hline AHI events $\cdot h^{-1}$ & $43.5 \pm 4.6(6.2-111.7)$ & $44.4 \pm 4.1(2.7-82.8)$ \\
\hline Nadir $\mathrm{Sa}, \mathrm{O}_{2} \%$ & $82.4 \pm 1.2(65-93)$ & $80.6 \pm 1.8(59-97)$ \\
\hline History of oral-facial surgery $\%$ & 17 & 33 \\
\hline History of allergic rhinitis $\%$ & 37 & 50 \\
\hline \multicolumn{3}{|l|}{ History of nasal stuffiness ${ }^{+} \%$} \\
\hline Never & 9 & 10 \\
\hline Occasional & 37 & 37 \\
\hline Frequent & 54 & 53 \\
\hline \multicolumn{3}{|l|}{ History of runny nose ${ }^{+} \%$} \\
\hline Never & 6 & 21 \\
\hline Occasional & 77 & 72 \\
\hline Frequent & 17 & 7 \\
\hline \multicolumn{3}{|l|}{ History of sore throat ${ }^{+} \%$} \\
\hline Never & 27 & 17 \\
\hline Occasional & 50 & 55 \\
\hline Frequent & 23 & 28 \\
\hline Nasal steroids use ${ }^{+} \%$ & 46 & 40 \\
\hline
\end{tabular}

Data are presented as mean \pm SEM (range), $\mathrm{n}$ or the percentage of subjects within the treatment group. M: male; F: female; BMI: body mass index; ESS: Epworth Sleepiness Scale; AHI: apnoea-hypopnoea episodes per hour of sleep; Nadir $\mathrm{Sa}_{2} \mathrm{O}_{2}$ : lowest oxygen saturation during sleep. ${ }^{\#}$ : between group comparisons with unpaired t-test and Chi-squared test (all $\mathrm{p}$-values $>0.05$ ): $\because$ : score ranges from $0-24$, where 24 is the worst score attainable; ${ }^{+}$: symptoms in the last 12 months.

data, did not demonstrate any significant differences, except for body mass index (31.3 \pm 0.86 (missing data subjects) versus $\left.35.7 \pm 0.83 \mathrm{~kg} \cdot \mathrm{m}^{-2} ; \mathrm{p}=0.002\right)$.

Total inspiratory NAR, performed in the seated and supine positions (both $\mathrm{pm}$ ) prior to nCPAP application, were not significantly different between placebo and heated humidification groups (table 2; fig. 1). Similarly, measurements taken the following morning (supine; am) and the difference between the morning relative to evening (before and after nCPAP) measurements, calculated as a percentage change (am-pm $\%=\mathrm{am}-\mathrm{pm} / \mathrm{pm} \times 100)$, were not significantly different between groups (fig. 1). Analysis of the expiratory NAR data provided similar findings (table 2).

\section{CPAP pressure}

The mean effective CPAP levels for the two groups were not significantly different (table 2).

\section{Nasosinus symptoms}

A total of 64 subjects (94\%) completed the questionnaire the following morning. Of these, $92 \%$ reported experiencing at least one upper airway symptom during the night. The frequency of reported nasal stuffiness, discharge, burning, sore throat and dry mouth in the placebo group were $71 \%$, $32 \%, 41 \%, 47 \%$ and $82 \%$, respectively (fig. 2). This was similar and not significantly different to the humidification group (all Chi-squared statistic p-values $>0.05$ ). Also, the severity rated on VAS for each symptom and the composite score were not significantly different between the groups (table 2). In general, the symptoms were mild with the majority $(>88 \%)$ of responses being rated $\leqslant 5 \mathrm{~cm}$ on a $10 \mathrm{~cm}$ VAS.

\section{Subjective response}

Over one-third of the subjects in this study reported an improvement in sleep quality and feeling refreshed with nCPAP (fig. 3), with no differences seen between treatment groups (Chi-squared statistic; $\mathrm{p}>0.05$ ). The majority were able to tolerate nCPAP ( $\leqslant 10 \%$ reported poor tolerability) and, after the one night experience, $>80 \%$ of subjects predicted that their use of the device would be on a frequent-to-regular basis. Again, the ratings for these responses were comparable between groups (Chi-squared statistic; $\mathrm{p}>0.05$ ). No subjects who completed the titration study refused nCPAP therapy.

\section{Post hoc subgroup analyses}

Whilst the above results showed no benefit from heated humidification, there remained the possibility that some subgroups may benefit. Therefore, subgroup analyses were performed on subjects who reported, at baseline, a) "frequent or regular nasal stuffiness" $(n=40) ; b)$ high inspiratory NAR (i.e. above the group median $>0.20 \mathrm{kPa} \cdot \mathrm{L}^{-1} \cdot \mathrm{s}^{-1} ; \mathrm{n}=26$ ); and c) high CPAP $\left(>10 \mathrm{cmH}_{2} \mathrm{O} ; \mathrm{n}=24\right)$. In none of these groups was any significant benefit shown for humidification versus placebo, on morning nasal symptoms, NAR and subjective responses, including tolerability and acceptability of treatment.

\section{DISCUSSION}

The findings from the present study indicate that the use of heated humidification during the initial titration study of nCPAP offers no additional benefit with respect to NAR, nasal symptoms, therapeutic CPAP level, subject response and the acceptance of therapy. This is in agreement with the only other randomised controlled study, which has examined the effects of heated humidification administered at the time of nCPAP initiation [21]. Contrary to these observations, the application of heated humidification in patients selected for chronic nasal/ oral symptoms has been shown to improve nasal symptoms, patient comfort and compliance [12, 22, 23]. Even among unselected groups of patients, such as those included in the current study, heated humidification given over a longer duration ( $\geqslant 3$ weeks) has been reported to reduce the incidence and severity of nasopharyngeal symptoms [13, 14, $24,25]$. However, in the latter case, the effect on nCPAP compliance and patient satisfaction has not consistently been in favour of heated humidification over placebo.

There are several potential reasons for the lack of positive findings in the present study. First, the application of heated humidification for one night may be insufficient to derive the benefits seen with more regular use. Systematic use of heated humidification over longer periods of time have been shown by other studies to offer advantages on patient comfort, acceptance and compliance to nCPAP $[13,14,24,25]$. Secondly, the severity of nasopharyngeal symptoms in the subjects presented here was mild with generally low mean 


\begin{tabular}{|c|c|c|c|}
\hline Variables & Placebo & Heated humidification & p-value \\
\hline \multicolumn{4}{|l|}{ Total inspiratory NAR $\mathrm{kPa} \cdot \mathrm{L}^{-1} \cdot \mathrm{s}^{-1}$} \\
\hline Seated: pm & $0.32 \pm 0.06(0.1-1.61)$ & $0.30 \pm 0.04(0.08-0.83)$ & 0.89 \\
\hline Supine: pm (before nCPAP) & $0.36 \pm 0.09(0.06-2.5)$ & $0.33 \pm 0.09(0.02-2.10)$ & 0.66 \\
\hline am-pm difference $\%$ & $278 \pm 221(-81-5281)$ & $27.5 \pm 13.3(-93-139.5)$ & 0.32 \\
\hline \multicolumn{4}{|l|}{ Total expiratory NAR $\mathrm{kPa} \cdot \mathrm{L}^{-1} \cdot \mathrm{s}^{-1}$} \\
\hline Seated & $0.31 \pm 0.03(0.07-0.8)$ & $0.38 \pm 0.06(0.07-1.2)$ & 0.46 \\
\hline Supine: pm (before nCPAP) & $0.38 \pm 0.06(0.05-1.4)$ & $0.53 \pm 0.27(0.07-5.9)$ & 0.85 \\
\hline Supine: am (after nCPAP) & $0.41 \pm 0.10(0.12-2.7)$ & $0.34 \pm 0.05(0.07-1.0)$ & 0.77 \\
\hline Effective CPAP $\mathrm{cmH}_{2} \mathrm{O}$ & $9.95 \pm 0.58(6.0-18.5)$ & $10.16 \pm 0.74(4.0-19.0)$ & 0.91 \\
\hline Sore throat & $0.68 \pm 0.19(0.0-4.2)$ & $1.40 \pm 0.38(0.0-9.3)$ & 0.15 \\
\hline Dry throat & $2.45 \pm 0.38(0.0-8.5)$ & $3.88 \pm 0.54(0.0-9.3)$ & 0.23 \\
\hline Composite VAS (worse $=50 \mathrm{~cm}$ ) & $7.20 \pm 1.02(0.0-19.5)$ & $8.95 \pm 1.27(0.0-24.6)$ & 0.48 \\
\hline
\end{tabular}

Data presented as mean \pm SEM (range). NAR: nasal airway resistance; nCPAP: nasal continuous positive airway pressure; VAS: visual analogue score on a $10 \mathrm{~cm}$ scale $($ very severe $=10 \mathrm{~cm})$ am- $\mathrm{pm}$ difference $\%:(\mathrm{am}-\mathrm{pm}) / \mathrm{pm} \times 100$. All $\mathrm{p}$-values were obtained using unpaired t-test after log transformation of data.

scores in the placebo group. This may be related to the relatively brief exposure to $\mathrm{nCPAP}$, which in this case was a single night application compared with longer exposures in patients already established on treatment. In the current scenario, any improvement in symptom severity from humidification (i.e. the effect size) would be expected to be quantitatively small and, therefore, may require a larger

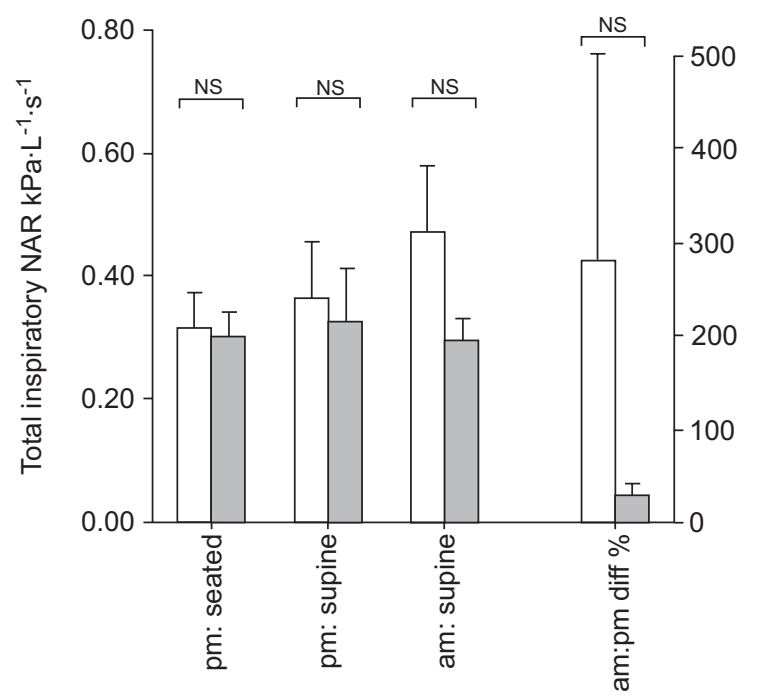

FIGURE 1. Total inspiratory nasal airway resistance (NAR) measured at different time points: pm before nasal continuous positive airway pressure (nCPAP) in the seated and supine positions; the next am after nCPAP; and the percentage increase in am relative to $\mathrm{pm}$ value for placebo and humidification groups. Data are expressed as mean \pm SE am-pm difference (diff. \%) $=(\mathrm{am}-\mathrm{pm}) / \mathrm{pm} \times 100 . \quad \square$ : placebo; $\square:$ : humidifier. NS: nonsignificant. sample size to detect a difference. In contrast, prolonged nCPAP use may give rise to more severe symptoms, where a greater benefit may be obtained from heated humidification. Indeed, if the current authors had examined the effect of humidification at a later time point or with longer nCPAP use, the findings might have been different. However, the focus of the present study was to address the effects of humidification during nCPAP therapy initiation; the motivation being that this strategy may potentially reduce the reported 20-42\% refusal rate that follows the first night titration, for reasons

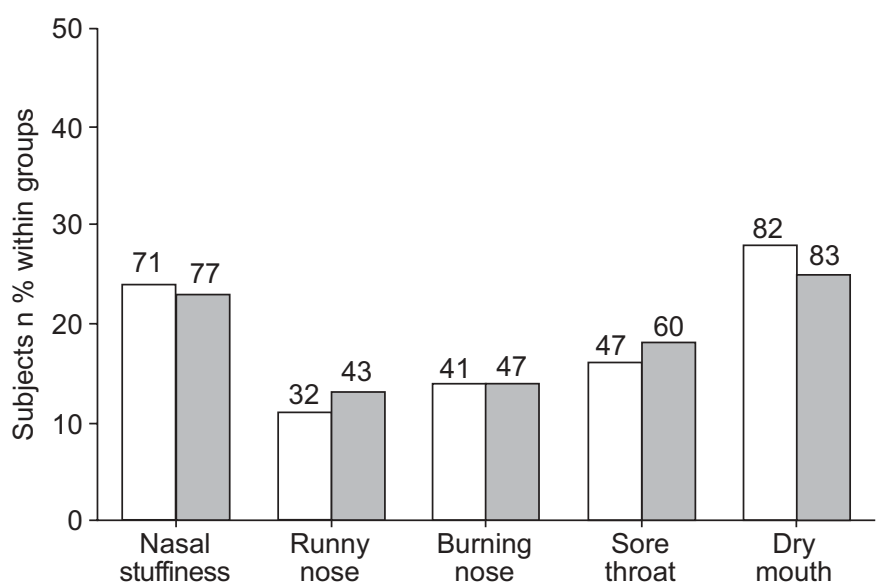

FIGURE 2. Number of subjects (presented as the percentage within each group) reporting nasopharyngeal symptoms related to nasal continuous positive airway pressure by treatment groups. $\square$ : placebo; $\square$ : humidifier. Comparisons between proportions of subjects were performed using the Chi-squared test. All p-values $>0.05$. 
relating to nasal and mask intolerance [5, 26, 27]. Furthermore, anecdotal evidence suggests that subsequent trials with nCPAP following an unsuccessful first attempt often lead to further failure [26]. Finally, it is possible that the scales and the questionnaire used may lack sensitivity. This was considered unlikely, as similar scales and questionnaires have been used in other studies with positive results [13, 14, 24].

The relationship between the acceptance and adherence of nCPAP to adverse nasopharyngeal symptoms has previously been examined. Earlier studies which relied on patientadministered questionnaires were unable to demonstrate a correlation between the prevalence of nasal symptoms and the rates of nCPAP acceptance [26, 28]. The presented results are in keeping with these findings. However, patients who refused or eventually stopped therapy were reported to give nasal symptoms, along with other reasons, as a cause for treatment discontinuation $[26,27]$. Without the consideration of potential confounders, such as OSAS severity, comorbidity, co-interventions, and patient's psychosocial background, this may have led to conclusions that overestimated the causal role of nasal symptoms in the refusal of therapy. Studies that have examined the relationship between nasal symptoms and nCPAP compliance have reported conflicting results. Using inbuilt CPAP monitors to quantify compliance and the pattern of use, some studies have demonstrated similar rates of nasal/
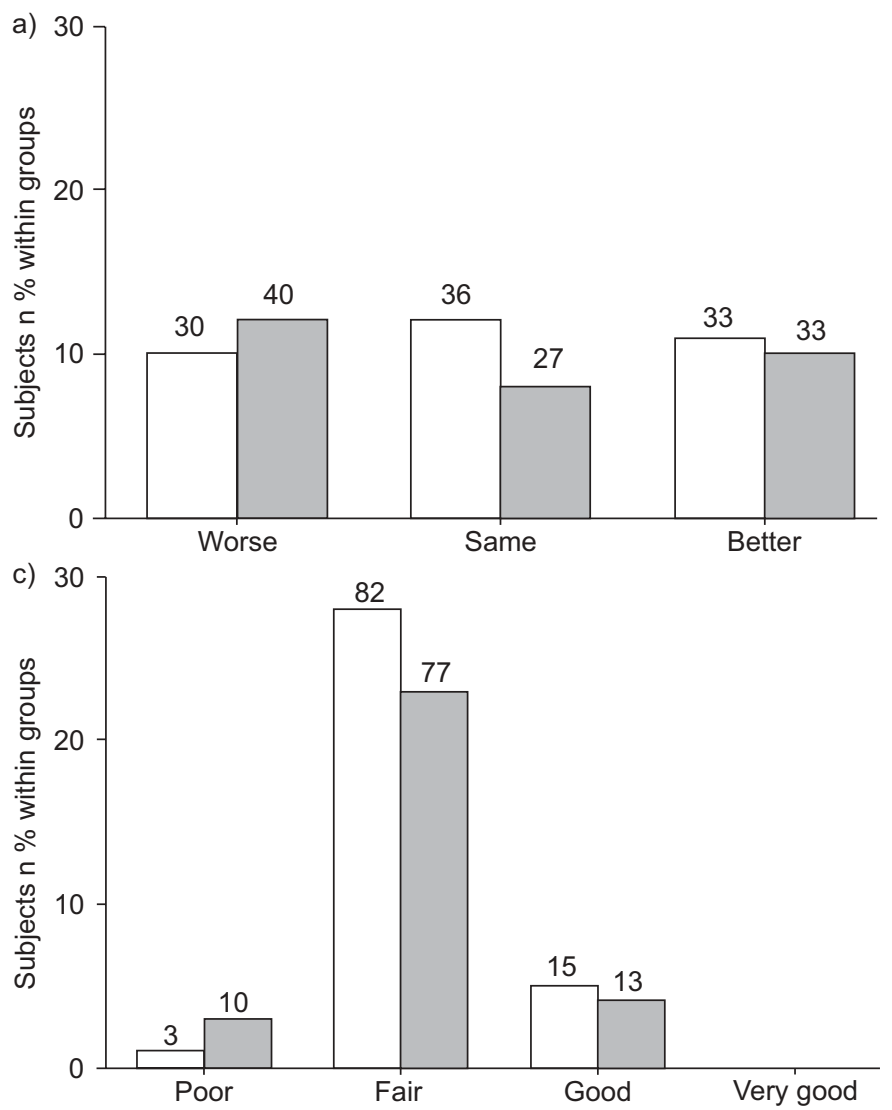

oral side-effects between compliant $\left(>4 \mathrm{~h} \cdot\right.$ night $\left.^{-1}\right)$ and less compliant patients $[7,10]$. In contrast, another study showed that patients who complained of nasal problems tended to use nCPAP less than those who reported no problems [9]. Furthermore, heated and cold pass-over humidification have been shown to be equally efficacious in reducing nasal symptoms, and yet have varying effects on nCPAP compliance $[13,14,25]$. These inconsistencies across different studies suggest that the relationship between nasal symptoms and compliance is unlikely to be straightforward. It needs to be considered in a framework that takes into account the dynamic interplay between physical and psychosocial factors. Although the present results are more relevant to the issue of therapy acceptance, some of the findings are informative regarding likely future therapy adherence, as it reflects on the patient's level of motivation and attitude towards therapy.

In the current cohort of untreated OSAS subjects, up to $92 \%$ reported experiencing at least one nasopharyngeal symptom during the night. This rate is higher than that expected for long-term nCPAP users [7, 10, 27, 28] and may reflect the difference in the current population and study design. Few studies have provided details of pre-existing nasal conditions prior to the commencement of nCPAP [7, 10, 27-29]. Half of the subjects in the present study indicated a history of allergic rhinitis and having experienced frequent nasal symptoms in

b)

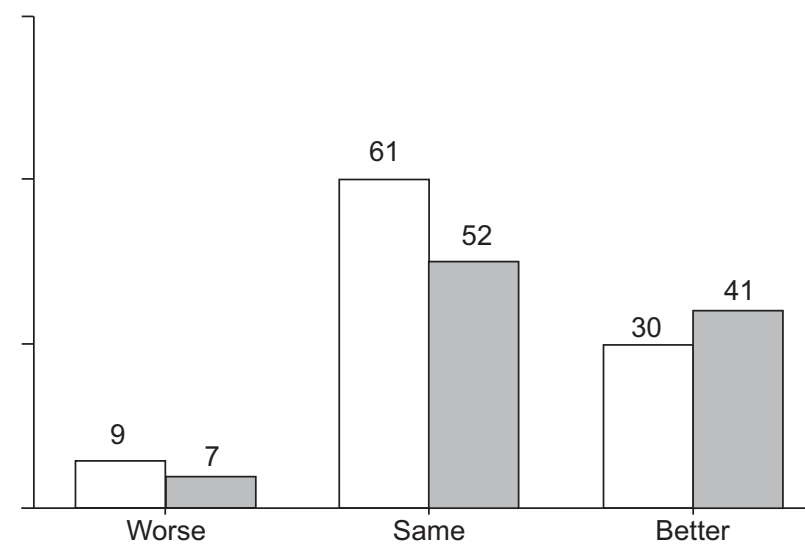

d)

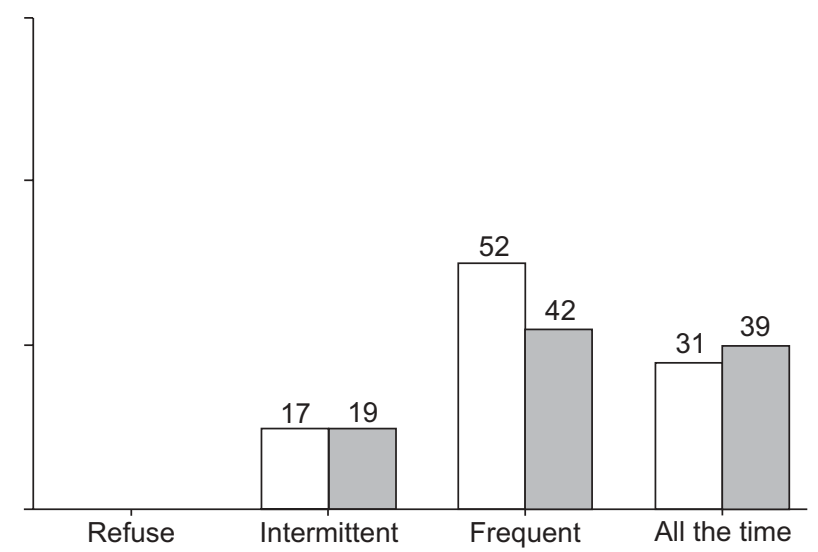

FIGURE 3. Number of subjects (presented as the percentage within groups) rating response to nasal continuous positive airway pressure (nCPAP) for a) sleep quality, b) feeling of freshness on awakening, c) nCPAP tolerance and d) predicted use of nCPAP. $\square$ : placebo; 1 : humidifier. Comparisons between groups was performed using the Chi-squared test. All p-values $>0.05$. 
the past year. Disorders of the upper respiratory tract may increase the risk and severity of OSAS $[30,31]$ and, similarly, may contribute to the development of adverse symptoms with nCPAP [32]. Furthermore, the present authors administered the questionnaire immediately following nCPAP, which may have led to a higher rate of positive response compared with studies that have relied on patient recall over a period of time.

Whilst the overall prevalence of nasopharyngeal symptoms was high, the severity was mild and did not appear to adversely impact on subject tolerability or prediction of nCPAP use. Direct comparison of the current results to previous studies is difficult due to the paucity of data pertaining to the severity of nasal symptoms. However, based on reported percentages of patients requiring treatment for nasopharyngeal side-effects, the prevalence of distressing symptoms can be estimated to be $38-56 \%$ amongst long-term nCPAP users $[23,29]$. In contrast, the authors found $<20 \%$ of the responses being rated $\geqslant 5$ on VAS (10 being the most severe) in this study. As previously mentioned, it is probable that a single night of nCPAP may result in milder symptoms, whereas higher rates of distressing symptoms are more likely associated with prolonged use.

In subjects with and without OSAS, the presence of mouth leaks during nCPAP leads to an excessive drying of the nasal mucosa, increase in NAR, release of inflammatory mediators and the development of upper airway symptoms. Heated humidification significantly attenuates these responses and alleviates symptoms $[15,22,33]$. In the present study, NAR tended to rise following overnight $\mathrm{nCPAP}$, particularly in the placebo group. However, the difference in morning NAR did not reach statistical significance between the groups. Similarly, the mean NAR change with nCPAP was higher in the placebo group compared with the heated humidification group (278\% versus $28 \%$ ), but this result was heavily influenced by three subjects who experienced major rises in NAR. By their nature, NAR measurements are widely variable and large sample sizes are likely required to detect a small-to-moderate effect of humidification. Based on a night-to-morning coefficient of variation of NAR in the order of $40 \%$, the current authors estimated that a sample size of 48 patients was sufficient to detect a real difference of $33 \%$ between treatment arms (80\% power; twotailed level 0.05). Differences of this magnitude or less would be unlikely to be of clinical significance. Consequently, the present authors believe that the absence of a NAR difference between groups is unlikely to reflect a type-II error. Furthermore, the rise in NAR that has been reported with nCPAP in the presence of mouth leaks $[15,22,33]$ under experimental conditions has not been confirmed in unselected groups of patients in the setting of acute [34] or chronic nCPAP application [35]. Perhaps the practice of applying chinstraps in cases of suspected mouth leaks in the current study may have significantly affected the severity and proportion of subjects who would have otherwise had an adverse response to nCPAP.

While no benefit could be demonstrated for heated humidification in the overall group, subgroup analyses similarly did not confirm a benefit for subjects with significant pre-existing nasal symptoms, increased baseline NAR or those requiring high CPAP (possibly predisposing them to mouth leaks and more naso-sinus symptoms). However, it must be acknowledged that smaller sample sizes in these groups increase the risk of a type-II statistical error.

In conclusion, the present authors found, in a group of unselected patients presenting for nasal continuous positive airway pressure initiation, a high rate of reported mild upper airway symptoms both preceding and following a single night of nasal continuous positive airway pressure treatment. Despite the relatively high frequency of nasopharyngeal symptoms, the application of heated humidification during the initial nasal continuous positive airway pressure titration did not confer any benefit over placebo in regards to nasal physiology, nasal/oral adverse events, patient tolerance and attitude to nasal continuous positive airway pressure therapy. The presented results do not support the routine use of heated humidification during first night nasal continuous positive airway pressure titration studies and suggest that, in instances where this might already be routine practice, significant savings in technicians' time might be possible.

\section{REFERENCES}

1 Malhotra A, Ayas NT, Epstein LJ. The art and science of continuous positive airway pressure therapy in obstructive sleep apnea. Curr Opin Pulm Med 2000; 6: 490-495.

2 Stepnowsky CJ, Dimsdale JE. Dose-response relationship between CPAP compliance and measures of sleep apnea severity. Sleep Med 2002; 3: 329-334.

3 Drake CL, Day R, Hudgel D, et al. Sleep during titration predicts continuous positive airway pressure compliance. Sleep 2003; 26: 308-311.

4 Sin DD, Mayers I, Man GC, Pawluk L. Long-term compliance rates to continuous positive airway pressure in obstructive sleep apnea: a population-based study. Chest 2002; 121: 430-435.

5 Grunstein RR. Nasal continuous positive airway pressure treatment for obstructive sleep apnoea. Thorax 1995; 50: 1106-1113.

6 Zozula R, Rosen R. Compliance with continuous positive airway pressure therapy: assessing and improving treatment outcomes. Curr Opin Pulm Med 2001; 7: 391-398.

7 Pepin JL, Leger P, Veale D, Langevin B, Robert D, Levy P. Side effects of nasal continuous positive airway pressure in sleep apnea syndrome. Study of 193 patients in two French sleep centers. Chest 1995; 107: 375-381.

8 Reeves-Hoche MK, Meck R, Zwillich CW. Nasal CPAP: an objective evaluation of patient compliance. Am J Respir Crit Care Med 1994; 149: 149-154.

9 Engleman HM, Martin SE, Douglas NJ. Compliance with CPAP therapy in patients with the sleep apnoea/hypopnoea syndrome. Thorax 1994; 49: 263-266.

10 Kribbs NB, Pack AI, Kline LR, et al. Objective measurement of patterns of nasal CPAP use by patients with obstructive sleep apnea. Am Rev Respir Dis 1993; 147: 887-895.

11 Waldhorn RE, Herrick TW, Nguyen MC, O'Donnell AE, Sodero J, Potolicchio SJ. Long-term compliance with nasal continuous positive airway pressure therapy of obstructive sleep apnea. Chest 1990; 97: 33-38.

12 Wiest GH, Lehnert G, Bruck WM, Meyer M, Hahn EG, Ficker JH. A heated humidifier reduces upper airway 
dryness during continuous positive airway pressure therapy. Respir Med 1999; 93: 26.

13 Massie CA, Hart RW, Peralez K, Richards GN. Effects of humidification on nasal symptoms and compliance in sleep apnea patients using continuous positive airway pressure. Chest 1999; 116: 403-408.

14 Neill AM, Wai HS, Bannan SP, Beasley CR, Weatherall M, Campbell AJ. Humidified nasal continuous positive airway pressure in obstructive sleep apnoea. Eur Respir J 2003; 22: $258-262$.

15 Richards GN, Cistulli PA, Gunnar Ungar R, BerthonJones M, Sullivan CE. Mouth leaks with nasal continuous positive airway pressure increases nasal airway resistance. Am J Respir Crit Care Med 1996; 154: 186.

16 Popescu G, Latham M, Allgar V, Elliott MW. Continuous positive airway pressure for sleep apnoea/hypopnoea syndrome: usefulness of a 2 week trial to identify factors associated with long term use. Thorax 2001; 56: 727-733.

17 McArdle N, Devereux G, Heidarnejad H, Engleman HM, Mackay TW, Douglas NJ. Long-term use of CPAP therapy for sleep apnea/hypopnea syndrome. Am J Respir Crit Care Med 1999; 159: 1108-1114.

18 Weaver TE, Kribbs NB, Pack AI, et al. Night-to-night variability in CPAP use over the first three month of treatment. Sleep 1997; 20: 278-283.

19 Rechtschaffen A, Kales A. A manual of standardized terminology, techniques and scoring systems for deep states of human subjects. Washington DC, US Printing Office, 1968. NIH Publication No. 204.

20 Clement PAR. Committee report on standardization of rhinomanometry. Rhinology 1984; 22: 151-155.

21 Wiest GH, Harsch IA, Fuchs FS, et al. Initiation of CPAP therapy for OSA: does prophylactic humidification during CPAP titration improve initial patient acceptance and comfort. Respiration 2002; 69: 406-412.

22 Martins de Araujo MT, Vieira SB, Vasquez EC, Fleury B. Heated humidification or face mask to prevent upper airway dryness during continuous positive airway pressure therapy. Chest 2000; 117: 142-147.

23 Rakotonanahary D, Pelletier-Fleury N, Gagnadoux F, Fleury B. Predictive factors for the need for additional humidification during nasal continuous positive airway pressure therapy. Chest 2001; 119: 460-465.
24 Worsnop CJ, Miseski S, Rochford P. The routine use of humidification with nasal CPAP for sleep apnoea. Intern Med J 2004; 3: A22.

25 Kline LR, Carlson P. NCPAP acceptance and compliance is altered by humidification. Sleep 1999; 22: Suppl., S230.

26 Rauscher H, Popp W, Wanke T, Zwick H. Acceptance of CPAP therapy for sleep apnea. Chest 1991; 100: 1023.

27 Engleman HM, Asgari-Jirhandeh N, McLeod AL, Ramsay CF, Deary IJ, Douglas NJ. Self-reported use of CPAP and benefits of CPAP therapy: a patient survey. Chest 1996; 109: 1470-1476.

28 Hoffstein V, Viner S, Mateika S, Conway J. Treatment of obstructive sleep apnea with nasal continuous positive airway pressure. Patient compliance, perception of benefits, and side effects. Am Rev Respir Dis 1992; 145: 841-845.

29 Sanders MH, Gruendl CA, Rogers RM. Patient compliance with nasal CPAP therapy for sleep apnea. Chest 1986; 90: 330-333.

30 McNicholas WT, Tarlo S, Cole P, et al. Obstructive apneas during sleep in patients with seasonal allergic rhinitis. Am Rev Respir Dis 1982; 126: 628.

31 Staevska MT, Mandajieva MA, Dimitrov VD. Rhinitis and sleep apnea. Curr Allergy Asthma Rep 2004; 4: 193-199.

32 Winck JC, Delgado JL, Almeida JM, Marques JA. Heated humidification during nasal continuous positive airway pressure for obstructive sleep apnea syndrome: objective evaluation of efficacy with nasal peak inspiratory flow measurements. Am J Rhinol 2002; 16: 175-177.

33 Hayes MJ, McGregor FB, Roberts DN, Schroter RC, Pride NB. Continuous nasal positive airway pressure with mouth leak: effect on nasal mucosal blood flux and nasal geometry. Thorax 1995; 50: 1182.

34 Desfonds P, Planes C, Fuhrman C, Foucher A, Raffestin B. Nasal resistance in snorers with or without sleep apnea: effect of posture and nasal ventilation with continuous positive airway pressure. Sleep 1998; 21: 625-632.

35 Bossi R, Piatti G, Roma E, Ambrosetti U. Effects of longterm nasal continuous positive airway pressure therapy on morphology, function, and mucociliary clearance of nasal epithelium in patients with obstructive sleep apnoea syndrome. Laryngoscope 2004; 114: 1431-1434. 